\section{Prácticas de revisión y edición de textos de alumnos bilingües indígenas de licenciatura en Oaxaca: la reflexividad y la intencionalidad en la redacción de comentarios Practices in the revision and editing of texts of bilingual indigenous students of licentiate courses in Oaxaca: reflection and intention in the forming of commentaries}

Ernesto Hernández Rodríguez*
Resumen: Esta investigación aporta un estudio exploratorio de las prácticas de revisión y edición de los textos redactados por 26 estudiantes bilingües indígenas de la licenciatura en docencia, quienes, además, son profesores de educación básica en distintas comunidades de Oaxaca, México. Los alumnos leyeron un texto sobre el tema del relativismo lingüístico y se les pidió que escribieran sus puntos de vista. Asumieron el rol de jueces y revisaron el texto de uno de sus compañeros. Les escribieron comentarios y sugerencias, con el fin de que el autor modificara su texto para mejorarlo. Los textos redactados, los comentarios de los jueces y las modificaciones realizadas en las segundas versiones permiten estudiar la intencionalidad en las decisiones de textualización durante la edición y la reflexividad del alumno sobre esta práctica de redacción. Los alumnos tienen dificultad para redactar sus puntos de vista, abordar el tema de la lectura y no desviarse del mismo. Se hace una caracterización del tipo de sugerencias de los jueces y las decisiones de textualización del autor al modificar su texto. Los criterios para editar el texto son complejos y reflejan regulación, intencionalidad y reflexividad, y no se limitan a lo planteado por los jueces.

Palabras clave: Reflexividad; Intencionalidad; Redacción.

Abstract: This investigation brings an exploratory study of the practices of revision and editing of texts written by 26 indigenous bilingual
* Licenciado en Lingüística por la Universidad Autónoma Metropolitana Iztapalapa. Maestro en Ciencias con especialidad en investigaciones educativas por el Centro de Investigación y de Estudios Avanzados del Instituto Politécnico Nacional. Desarrolla investigación sobre la reflexividad en los procesos de redacción en el proyecto "Dominio lingüístico y académico de alumnos bilingües indígenas en algunas universidad de México" en el Doctorado en Lingüística de la UAM Iztapalapa. E-mail: ernestotem@live.com.mx
Tellus, ano 11, n. 21, p. 71-96, jul./dez. 2011

Campo Grande, MS 
students taking a teacher training course who are also basic education teachers in distinct communities in Oaxaca, Mexico. The students read a text on the theme of linguistic relativism and they were asked to write their points of view. They took on the role of judges and revised the text of one of their companions. They wrote commentaries and suggestions, with the intention that the author should modify their text in order to improve it. The written texts, the commentaries of the judges and the modifications carried out on the second versions permitted the study of intention in the decisions of the textualization during editing and reflection of the students on the practice of writing. The students had difficulty in writing their points of view, approaching the theme of the reading and in going off the subject. There was a similarity in the type of suggestions given by the judges and the decisions of textualization of the author in modifying their texts. The criteria for the editing of the text are complete and show regularization, intention and reflection, and are not limited to that indicated by the judges.

Key words: Reflection; Intention; Composition.

\section{Problemáticas de la redacción en nivel superior}

Uno de los problemas a los que se enfrentan los estudiantes universitarios es reflejar de forma escrita los conocimientos adquiridos. La realidad es que en nuestros ambientes académicos en la educación superior la redacción no se concibe como un elemento fundamental para la construcción gradual de conocimiento en las distintas disciplinas. Esta situación sobre el papel de la redacción en el nivel superior se presenta en todos los contextos, tanto en alumnos bilingües o monolingües, indígenas o no indígenas. Es una realidad que caracteriza al sistema educativo actual en México. En muchos casos, se considera que los alumnos que llegan a la licenciatura ya saben redactar y el docente se dedica exclusivamente a su disciplina, de manera tal que el alumno se las arregla como puede para resolver las problemáticas que implica responder a las exigencias académicas de redacción.

En general, los productos de los estudiantes presentan múltiples problemáticas sobre la textualización, misma que incluye el manejo de los recursos para plasmar por escrito las ideas. Así, considero que la textualización involucra la toma de decisiones, por ejemplo, sobre la estructuración, el lenguaje empleado, el contenido, la distribución de las ideas, la construcción de interlocutores, la coherencia, la cohesión, así como las valoraciones comunicativas y sociales sobre la pertinencia, la aceptabilidad y la eficiencia en el contexto del escrito. Los alumnos se enfrentan a la dificultad para concebir sus interlocutores y al reconocimiento de la diversidad de géneros textuales. Tienen que identificar el contexto de uso de los textos en ambientes académicos e interactuar en una comunicación escrita que en gran parte les es ajena. El alumno se enfrenta a un conjunto de dificultades y retos sobre los complejos procesos de textualización e interacción que implica la redacción de textos académicos. 


\section{Entorno lingüístico y educativo de Ixtepec, Oaxaca}

En la educación primaria en Oaxaca se imparte de manera regional la enseñanza de la lengua indígena como lengua materna o segunda lengua como parte de la educación oficial bilingüe. Sin embargo, en general, a lo largo de la historia en Oaxaca ha prevalecido una fuerte política de castellanización y relaciones complejas de desplazamiento de las lenguas indígenas en las funciones cotidianas básicas, además, actualmente existe un complejo contacto de lenguas y relaciones lingüísticas en el marco del intercambio comunicativo en la globalización y las tecnologías. En los niveles medio y medio superior la educación se imparte predominantemente en español, aunque existen algunos sistemas educativos que incorporan la enseñanza de las lenguas indígenas y las recientes propuestas de formación intercultural en la educación superior que incorporan la presencia de las diversas lenguas de la región, incluido, por supuesto, el español y lenguas extranjeras. A grandes rasgos, Oaxaca es uno de los estados con mayor pobreza del país, y esto se ve reflejado en las precarias condiciones educativas en la educación bilingüe indígena, principalmente, por el reducido número de docentes bilingües indígenas con formación universitaria y la deserción de los alumnos por las condiciones económicas.

En este trabajo presento los resultados de investigación sobre un ejercicio de prácticas de revisión y edición de textos en ambientes académicos con 26 estudiantes universitarios bilingües indígenas en la población de Ixtepec, correspondiente al distrito de Juchitán en la región del Istmo de Tehuantepec en el estado mexicano de Oaxaca en el Sureste de México ${ }^{1}$.

\footnotetext{
${ }^{1}$ Realicé este ejercicio en abril de 2011 como parte del trabajo conjunto que desarrollamos los integrantes en el Proyecto “Dominio lingüístico y académico de estudiantes bilingües indígenas en universidades de México: habilidades comunicativas y académicas, reflexividad, sociolingüística y políticas interculturales del lenguaje" a cargo del Dr. Héctor Muñoz Cruz, investigador del Doctorado en Lingüística de la Universidad Autónoma Metropolitana Iztapalapa.
} 


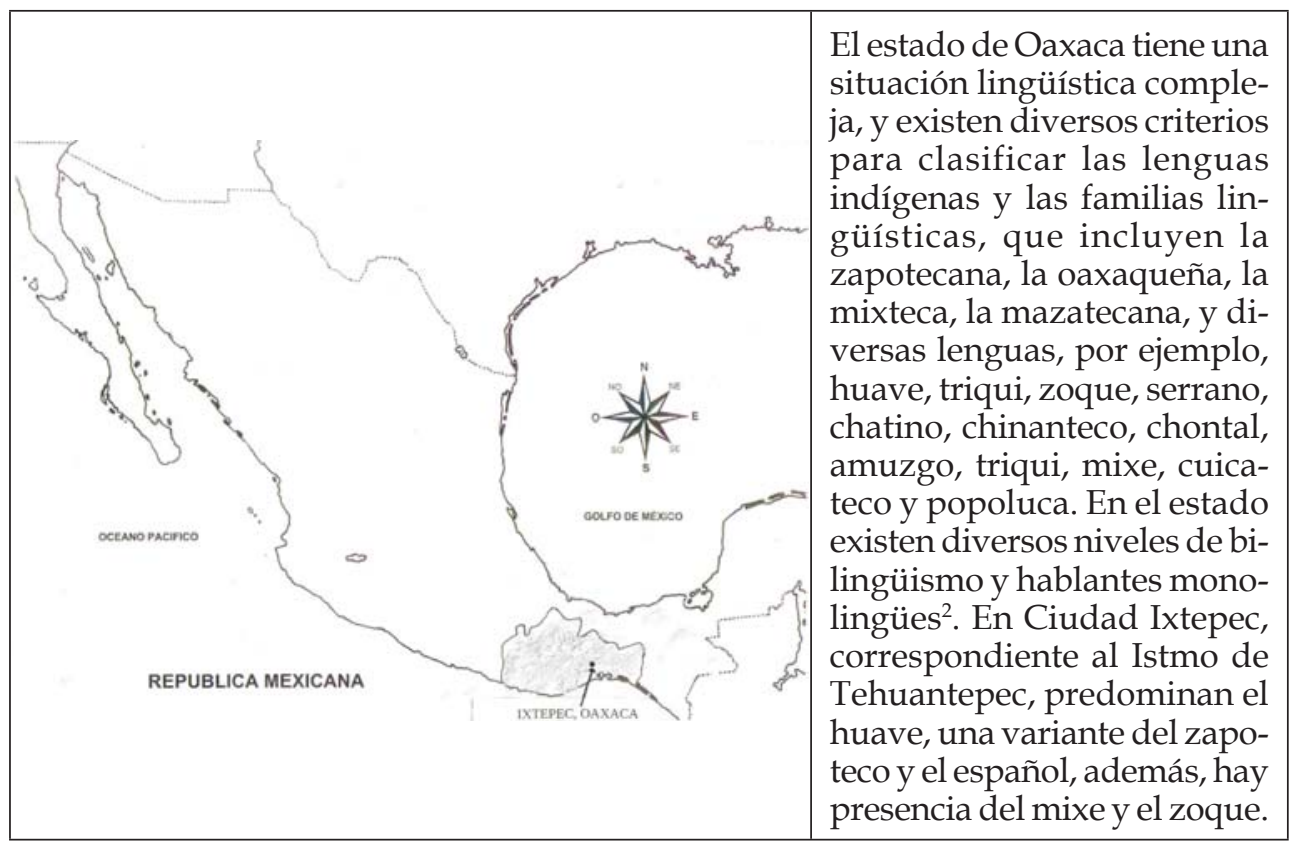

Los 26 estudiantes de la Universidad Pedagógica Nacional en la unidad Ixtepec asistían a un curso de formación de profesores bilingües indígenas de educación básica de distintas comunidades de Oaxaca ${ }^{3}$. Esta universidad imparte las licenciaturas en docencia indígena en nivel básico y ofrece capacitación docente. Actualmente estos alumnos, además de estudiar el nivel superior, desarrollan su trabajo docente en nivel primaria en comunidades con población mayoritariamente bilingüe indígena. Ante la escasez de profesores bilingües indígenas es común que los estudiantes de licenciatura en docencia comiencen a trabajar en sus comunidades al iniciar sus estudios. De hecho, muchos alumnos de nivel medio superior ya trabajan como instructores que son capacitados para el trabajo docente en nivel básico y posteriormente tienen la opción de cursar el nivel superior en docencia.

Los alumnos universitarios manifestaron tener distintos niveles de bilingüismo, todos hablan español y alguna de las siguientes lenguas: zapoteco, zoque, mixe, chatino y huave. De los 12 alumnos cuya lengua materna

\footnotetext{
${ }^{2}$ Para una revisión completa del panorama lingüístico y de los niveles de bilingüismo consultar Manrique Castañeda (1988) y Cifuentes (1998).

${ }^{3}$ Anteriormente, en México quienes deseaban ser profesores de nivel básico no requerían cursar licenciatura, y, de manera directa, al concluir la educación secundaria se incorporaban a la Escuela Normal Superior de Maestros, donde se formaban como docentes. Recientemente, se ha implantado e impulsado la licenciatura en docencia.
}

74 Ernesto H. RODRÍGUEZ. Prácticas de revisión y edición de textos de alumnos bilingües... 
es el español, ocho dijeron ser monolingües ${ }^{4} \mathrm{y}$ los otros cuatro tienen como segunda lengua la indígena, en tanto que 14 dijeron tener la lengua indígena como materna.

\section{Propósitos y alcances del ejercicio propuesto}

El propósito del ejercicio es hacer una descripción de las prácticas de redacción, revisión y edición de los textos que redactaron los estudiantes de nivel superior. El centro de interés era que los alumnos, asumieran el rol de jueces que revisarían el texto de uno de sus compañeros e hicieran comentarios y sugerencias por escrito, con el fin de que el autor modificara su texto con la idea de mejorarlo. Además de la descripción de recursos de textualización, me interesaba destacar a partir de los escritos de los alumnos en los textos comentados y editados, aquellos elementos de reflexividad sobre la experiencia de revisión, edición y lo expresado al asumir el papel de jueces al revisar y comentar los textos. La reflexividad sobre la textualización puede incluir aspectos diversos, tales como lo cognitivo, lo afectivo, la inseguridad, el prestigio, criterios sobre el desempeño lingüístico y las actitudes ante dificultades enfrentadas en la redacción.

El punto de interés radica en los recursos empleados en los procesos de escritura para dar cuenta de las diversas problemáticas y decisiones de textualización involucradas en la planeación y la redacción antes, durante y después de escribir el texto, así como las valoraciones expresadas por los alumnos al asumir el papel de jueces, $y$, por otra parte, la manera en que el autor contempla dichas observaciones para tomar decisiones en el proceso de textualización para editar su texto, con la intención de mejorarlo.

A pesar de que en los textos podemos apreciar que en su mayoría los estudiantes tienen dificultades para la redacción, evito hacer valoraciones normativas y prescriptivas o juicios valorativos sobre los recursos de redacción de los alumnos, tampoco pretendo hacer interpretaciones o explicaciones sobre las diversas causas del nivel de dominio de recursos de escritura; tan sólo me centro en dar cuenta de las prácticas y decisiones de los estudiantes en el manejo de la redacción durante la revisión y edición de los textos y los elementos de reflexividad plasmados por escrito en el ejercicio realizado al asumir el rol de juez y editar sus propios textos a partir de los comentarios y sugerencias recibidas.

Independientemente del dominio del español por parte de los alumnos cuya lengua materna es la indígena y que, en algunos casos, presentan

\footnotetext{
${ }^{4}$ Tomamos la decisión de considerar el bilingüismo incipiente y funcional para aquellos alumnos que manifestaban tener conocimientos de la lengua indígena y que podían entenderla en algunas interacciones y en algunos casos comunicarse de manera básica en su entorno cotidiano.
} 
evidencias de no tener un completo dominio del español, por ejemplo, en la estructuración gramatical y el vocabulario, en general contemplo que las prácticas de escritura académica y el tipo de decisiones de textualización y reflexividad son similares, ya sea la lengua indígena o el español la lengua materna ${ }^{5}$. Estos aspectos son propios del lenguaje escrito en ambientes académicos en todos los entornos lingüísticos, por lo que evito centrarme en hacer caracterizaciones sobre el nivel de desempeño o dominio del español de los alumnos indígenas por su condición de bilingüismo.

Considero que los enfoques centrados exclusivamente en la psicolingüística y la didáctica no logran contemplar los aspectos fundamentales sobre lo que vive, siente y considera el autor de un texto en los diversos planos involucrados en la textualización, precisamente porque estos enfoques corren el riesgo de basarse en modelos centrados en el análisis y la interpretación del investigador y en prescripciones a partir de diseños preestablecidos que no siempre se pueden aplicar en diversos contextos. Por ello, en relación a los alcances de esta investigación, me interesa hacer una propuesta etnográfica para estudiar la reflexividad de los individuos sobre la redacción, que aporte una perspectiva crítica, comunicativa e integral de la escritura en ambientes académicos.

\section{Revisión de estudios sobre el tema}

El estudio de la redacción en ambientes académicos en la educación superior desde una perspectiva de la etnografía en las prácticas cotidianas y la reflexividad es un campo que comienza a desarrollarse en los trabajos de Lea (1999), Lea e Street (1998), Scott (1999), Lillis (2001), Street (2009a, 2009b) y Zavala (2009). El enfoque etnográfico proporciona una herramienta metodológica fundamental para interactuar en situaciones que dan relevancia al contexto y la práctica, por ejemplo, en las propuestas de Brice Heath (1983) y Brice Heath e Street (2008).

El trabajo de Beugrande e Dressler (1997), además de ser un texto clásico fundamental precursor sobre el estudio de la textualización, nos es de utilidad debido al establecimiento de los principios constitutivos de cohesión, coherencia, intencionalidad, informatividad, situacionalidad y aceptabilidad, y

\footnotetext{
${ }^{5}$ De hecho, en mi trabajo sobre redacción argumentativa de alumnos monolingües hablantes del español de nivel medio superior, muestro que ante las dificultades enfrentadas para organizar un texto argumentativo y abordar el tema, los alumnos por momentos presentan problemáticas en la estructuración gramatical, por ejemplo, en las relaciones de referencia y concordancia de género y número. (Hernández Rodríguez, 2003). Esto pone de manifiesto que estas problemáticas no son exclusivas de los indígenas, en realidad es un asunto que corresponde al complejo proceso del desarrollo de la práctica escrita.
}

76 Ernesto H. RODRÍGUEZ. Prácticas de revisión y edición de textos de alumnos bilingües... 
por otra parte, los principios regulativos de eficacia, efectividad y propiedad. Contemplo esta propuesta y la relación entre reflexividad e intencionalidad como mecanismos básicos de la comunicación (Hyland, 2005; Recanati, 1979, 2004). De hecho Hyland desarrolla su concepto de reflexividad a partir del trabajo de redacción y las experiencias de textualización desde una perspectiva que construye la relación entre el autor y la representación que éste hace de sus interlocutores desde las prácticas concretas ${ }^{6}$.

Como propuesta para el trabajo de análisis de los textos y los recursos de textualización, principalmente, en torno a la coherencia y la cohesión, también considero los enfoques de Halliday y Hasan (1976) y Adam (2005), así como las propuestas de análisis textual de Blanche-Benveniste (1998), Béguelin (2002) y Hernández Rodríguez (2003). En relación a los estudios sobre prácticas de regulación, revisión y edición de textos en ambientes escolares, considero la investigación de Camps (2009).

\section{Diseño de las situaciones e interacciones con los alumnos}

El profesor del grupo nos permitió llevar a cabo la interacción con sus alumnos. Propuse como actividad la lectura de un texto expositivo de difusión informativa sobre la discusión de la teoría Sapir-Whorf, en torno al relativismo lingüístico, en el que se plantea que la gramática de la lengua determina el pensamiento y la forma de concebir el mundo de las personas ${ }^{7}$. Además contempla dos hipótesis; la primera, denominada fuerte, establece que la lengua del monolingüe determina completamente su forma de pensar, memorizar y clasificar la realidad. Por otra parte, la hipótesis débil considera que la lengua de un hablante tiene cierta influencia en la forma en que éste conceptualiza y memoriza la realidad a nivel semántico y que existen diferencias significativas en la forma en que dos hablantes de lenguas diferentes enfocan la realidad. El texto además presenta algunas evidencias empíricas que contemplan algunos experimentos realizados que parecen confirmar la hipótesis Sapir-Whorf, pero no proporciona evidencia contundente de que el lenguaje provee todos los patrones del pensamiento. El tema abordado en la lectura es polémico, pero seleccioné un texto expositivo breve, aparentemente sencillo, simplemente para despertar el interés sobre un asunto en torno al

\footnotetext{
${ }^{6}$ Recanati $(1979,2004)$ construye el concepto de reflexividad a partir de la propuesta la enunciación en torno a la dualidad de opacidad y transparencia del signo lingüístico, así como la construcción de significados. Hyland (2005) incorpora los conceptos de reflexividad e intencionalidad a los procesos de textualización como prácticas interactivas donde el autor del texto construye las relaciones intertextuales así como las relaciones con los interlocutores.

${ }^{7}$ El texto expositivo es una adaptación para fines de divulgación que realizo a partir del texto de Whorf (1964) y de la reflexión de Manrique Castañeda (1988) sobre la importancia de las lenguas para el conocimiento de las formas culturales.
} 
uso de la lengua, contemplando que los alumnos son también profesores que participan en interacciones y contextos de bilingüismo y ésta sería una oportunidad para reflexionar sobre aspectos vinculados con su práctica cotidiana.

En esta ocasión el diseño de la situación y la interacción fue muy sencilla, sin una conducción dirigida a determinados aspectos, simplemente les dijimos a los alumnos que leyeran atentamente el texto, y que hicieran un comentario crítico sobre el mismo, cuidando de hacerlo con sus propias palabras. Evitamos hacer valoraciones y comentarios que impactaran o influyeran los puntos de vista de los alumnos, y simplemente nuestras intervenciones orales estaban dirigidas a que se motivaran a escribir. Una vez que escribieron su texto, les pedimos que lo intercambiaran con un alumno que asumiría el papel de juez, éste tendría que hacer comentarios al texto del compañero para ayudarle a que mejorara su escrito. Posteriormente, los jueces regresaron los textos a sus autores y entonces les indicamos que a partir de las observaciones realizadas, modificaran su redacción y que escribieran una segunda versión contemplando las sugerencias.

\section{Clasificación y análisis de acuerdo al género textual y al tema}

a) Clasificación de los escritos conforme al género textual

Como primer criterio para iniciar el análisis llevé a cabo la transcripción $^{8}$ y la clasificación de los textos conforme al género textual empleado en la redacción, tomando en consideración que les pedimos a los alumnos que hicieran un comentario crítico de la lectura. Esperábamos que los estudiantes tomaran una postura o punto de vista sobre la discusión del relativismo lingüístico presentado en la Hipótesis de Sapir-Whorf, y que lo asumieran en el comentario, de manera tal que escribieran con intencionalidad argumentativa, sin embargo como podemos apreciar en la tabla 1, en su mayoría escribieron textos expositivos en las versiones inicial y final (17 de 26) en los que simplemente exponen, parafrasean o copian el contenido de la lectura o bien exponen un tema relacionado pero ajeno al contenido concreto del texto. En algunas de estas exposiciones los alumnos se enfrentan a dificultades en la comprensión de la lectura del texto y esto los lleva a tener problemáticas en el proceso de textualización en distintos aspectos. Mediante esta clasificación

\footnotetext{
${ }^{8}$ Como criterio metodológico para la transcripción respeto la escritura de los alumnos tal como aparece en sus escritos, sin hacer modificaciones ni correcciones de ortografía, segmentación o acentuación. Sin embargo, en este trabajo, por motivos de espacio, no sigo la línea gráfica ni las segmentaciones al final de reglón, únicamente contemplo el cambio de párrafo. Utilizo la siguiente nomenclatura para ubicar y nombrar los textos: $t$ (texto), $s$ (sapir-whorf), i (Ixtepec), h (hombre), m (mujer), número, vi (versión inicial), vf (versión final), j (juez).
} 
podemos percatarnos de algunos elementos de reflexividad en las observaciones de los jueces, fundamentalmente, al sugerir al autor del texto que escriba comentarios sobre la lectura.

Tabla 1 - Clasificación de los textos conforme al género

\begin{tabular}{|l|c|c|c|c|}
\hline Género textual & $\begin{array}{l}\text { En versión } \\
\text { inicial y } \\
\text { final }\end{array}$ & $\begin{array}{l}\text { Combinado co- } \\
\text { mentario versión } \\
\text { inicial y exposi- } \\
\text { tivo versión final }\end{array}$ & $\begin{array}{l}\text { Combinado exposi- } \\
\text { tivo versión inicial } \\
\text { y comentario ver- } \\
\text { sión final }\end{array}$ & Total \\
\hline Textos expositivos & 17 & - & - & 26 \\
\hline Comentarios & 5 & - & - & 5 \\
\hline $\begin{array}{l}\text { Textos con parte ex- } \\
\text { positiva y comentario }\end{array}$ & 0 & 2 & 2 & 4 \\
\hline & 22 & 2 & 2 & 26 \\
\hline
\end{tabular}

Resulta significativo que una gran mayoría de alumnos utilice el género expositivo cuando se les indica que redacten un comentario. A pesar de que los textos reflejan poco avance y escasos recursos en la redacción, no pretendo hacer valoraciones normativas ni descalificar la redacción de los alumnos, por el contrario mi interés es estudiar, caracterizar y analizar estos textos desde la perspectiva de los principios constitutivos y regulativos planteados por Beugrande e Dressler (1997). Por ejemplo, considero la intencionalidad y la aceptabilidad, es decir, determinar aquellas evidencias sobre lo que el alumno intenta expresar desde su repertorio de conocimientos y habilidades. También considero las valoraciones de aceptabilidad que pudiera plantear el juez que revisa y hace sugerencias sobre el texto, quien puede contemplar criterios regulativos de eficacia, efectividad y propiedad. Precisamente, por ello es fundamental que un compañero revise el texto, de manera tal que la reflexividad sobre la textualización, la interacción y la retroalimentación, le permitan, por ejemplo, al autor del texto considerar la distinción entre la redacción expositiva y el comentario.

Son relevantes las redacciones que en la primera versión presentan un género textual determinado y en la segunda cambien al otro, comentario o exposición según sea el caso (4 de 26). Considero que la manera en que los autores de estos textos contemplaron y valoraron los comentarios de los jueces, influyó de manera determinante para cambiar el género textual en la versión final. En el caso de los escritos cuya primera versión es un comentario y la segunda es un texto expositivo, por ejemplo, en el texto tsim06j, el juez considera que es necesario contemplar la totalidad de la lectura. 
- Compañera Se debe abarcar todo el texto.

- Cuando uno platica con el autor no debemos perder el punto clave. (tsim06j)

Es posible que al hacer esta consideración la alumna decida contemplar el contenido de la lectura y exponerlo, y al hacerlo deje de lado el comentario que antes había realizado.

En el caso del texto tsim16 el juez no hace sugerencias, simplemente expone una idea totalmente alejada de la lectura sobre la lengua y la realidad, e invita a la compañera a leer más. Es posible que esta actitud del juez lleve a la alumna a exponer el tema para mostrar que leyó, dejando de lado el comentario realizado.

LA LENGUA ES UNA EXPRESIÓN QUE COMUNICA ESTADOS DE ANIMOS ALEGRIA POR LA CUAL EL SER HUMANOSE RELACIONA CON LA REALIDAD DEL MUNDO.

HAY QUE LEER MAS COMPAÑERA. (tsim16j)

En los casos de los dos textos donde los alumnos escriben primero una versión expositiva y después un comentario, parece que los comentarios puntuales de los jueces sobre la necesidad de que el autor exprese sus puntos de vista influyen en el cambio al comentario en la versión final. Revisemos las observaciones de estos jueces.

-Por lo que note, no te diste el tiempo de leer el texto, no te preocupes a la mayoría nos paso. "no es cierto"

-Pienso que debes ser un poquito mas crítico con tígo. (tsih07j)

Tu hipótesis whorfiana que tu escribiste no habla de que lengua.

compañero que tu concides con el científico por que tienen las mismas ideas de acuerdo al tema.

falto una explicación mas clara como se relaciona en la sociedad. (tsih20j)

En este último ejemplo, parece que el juez hace ironía sobre la coincidencia entre el científico y su compañero, puesto que éste se limita a exponer el contenido.

Esta primera clasificación de los textos nos deja ver claramente que en este entorno académico las prácticas de redacción no favorecen de manera general la redacción del comentario crítico, parece que en este caso, de manera cotidiana las prácticas de redacción expositiva predominan sobre la redacción de comentarios. Considero que no basta conocer e identificar los géneros textuales, en este caso la distinción entre comentario crítico y exposición, además, es necesario que la práctica de redacción académica incorpore múltiples 
experiencias significativas y criticas para que el alumno desarrolle el hábito y el gusto para la redacción de comentarios con intencionalidad argumentativa. Indudablemente, diversos factores contextuales influyen en el desempeño de estos alumnos, por ejemplo, los planes y programas de estudio y las políticas educativas, sin embargo estos aspectos requieren una revisión contextual amplia y no son el motivo de esta investigación.

b) Relación entre los contenidos de la lectura y de los escritos de los alumnos

Un aspecto fundamental para caracterizar los textos y para contemplar los principios constitutivos y regulativos en el proceso de revisión y edición de los textos es el establecimiento de la relación entre el contenido del texto para leer y los escritos de los alumnos. Esta información nos permite contemplar, principalmente, en algunos de los textos que no se relacionan con la lectura o que se desvían del tema, la naturaleza de la intencionalidad en la redacción y las posibles problemáticas enfrentadas para la comprensión del texto proporcionado para leer o bien las dificultades para la redacción que dan lugar a problemas para lograr una expresión clara. Además, contemplo los casos donde los comentarios del juez llevan a los autores a la toma de decisiones para intentar incorporar o eliminar el tema de la lectura en sus comentarios.

Tabla 2 - Relación entre la redacción de los alumnos y el texto que leyeron

\begin{tabular}{|c|c|c|c|c|}
\hline Textos & $\begin{array}{l}\text { Sin relación con la } \\
\text { lectura }\end{array}$ & $\begin{array}{l}\text { Intenta abordar el } \\
\text { tema pero no cor- } \\
\text { responde o se des- } \\
\text { vía de la lectura }\end{array}$ & $\begin{array}{l}\text { Se relaciona com- } \\
\text { pletamente con la } \\
\text { lectura }\end{array}$ & Total \\
\hline $\begin{array}{l}\text { Autor en ver- } \\
\text { sión inicial }\end{array}$ & $\begin{array}{c}11 \\
\text { (textos: 04, 07, 09, 10, } \\
15,16,18,19,22,23,26) \\
\end{array}$ & $\begin{array}{c}4 \\
\text { (textos:13,14, 20,25) }\end{array}$ & \begin{tabular}{l}
\multicolumn{1}{c|}{11} \\
(textos: $1,2,3,5,6,8$, \\
$11,12,17,21,24)$
\end{tabular} & 26 \\
\hline $\begin{array}{l}\text { Autor en ver- } \\
\text { sión final }\end{array}$ & \begin{tabular}{l}
\multicolumn{1}{c|}{13} \\
(textos: 04, 07, 09, 10, \\
$12,15,16,18,19,20,22$ \\
$25,26)$
\end{tabular} & $\begin{array}{c}2 \\
\text { (textos: 13,14) }\end{array}$ & \begin{tabular}{l}
\multicolumn{1}{c|}{11} \\
(textos: 1, 2, 3,5, 6, 8, \\
$11,17,21,23,24)$
\end{tabular} & 26 \\
\hline
\end{tabular}

La tabla 2 pone de manifiesto la necesidad de cuestionarnos sobre el tipo de prácticas académicas que deberían favorecer que los alumnos puedan leer un texto y escribir sobre éste abordando el tema en cuestión. El mayor número de textos sin relación con el tema de la lectura y el hecho de que éstos aumenten en la versión final (de 11 a 13), y la reducción de los textos con intencionalidad por abordar el tema (de 4 a 2), nos muestra datos reveladores 
que pueden involucrar no sólo la falta de experiencias significativas cotidianas de este tipo de prácticas, sino también dificultades para la comprensión de la lectura y la práctica de la redacción.

\section{1) Sin relación con la lectura}

En la tabla 2 podemos apreciar que más de la mitad de alumnos escribieron textos totalmente ajenos a la lectura, de hecho algunos de los jueces destacan que el alumno no leyó o no puso atención a la lectura, tal como vimos anteriormente en el ejemplo tsih07j. Pero en otros casos el juez valora positivamente el desempeño de su compañero, a pesar de que éste escribió un texto totalmente ajeno a la lectura realizada, tal como apreciamos a continuación.

Yo le entendi al texto que una persona habla y expresa sus sentimientos de acuerdo a su manera de como percibe el mundo que lo rodea.

$\mathrm{Y}$ de que nosotros, como otros seres vivos tenemos diferentes habilidades, y cada una tiene su manera de reconocer e interpretar. (tshi19vi)

El alumno no comenta el texto y redacta tal como si estuviera haciendo un reporte de aquello que comprendió sobre la lectura. En este caso el juez recomienda incorporar información, el comentario, la conclusión, y además considera que el autor hizo un "buen análisis". Ante estos comentarios el autor modifica su texto al incorporar información de manera esquemática sobre la lectura, pero se mantiene alejado del tema abordado. Este tipo de ejemplos me llevó a caracterizar los comentarios de los jueces y su impacto en la redacción de las versiones finales.

2) Versión inicial sin relación y final relacionada con el tema

Destaca el caso en que en la versión inicial el autor escribe un texto sin relación alguna con la lectura y en la versión final redacta un texto relacionado completamente con el tema, tal como sucede en tsih23. En este caso el único elemento que hace referencia a la necesidad de escribir sobre el tema es el mencionar la falta de datos, por lo demás, el juez parece no aportar elementos para que el alumno haya realizado el cambio de tema.

La observación es que la critica de un parrafo ${ }^{9}$ no es solamente lo que nos muestra, solamente falta más datos Interesante, la escritura no dire que es buena ni mala porque la mía es peor, solamente modificarla un poco. [...] (tsih23j)

\footnotetext{
${ }^{9}$ A pesar de que el texto tsih23 está conformado por dos párrafos el juez considera que está conformado sólo por uno. Este tipo de ejemplos me lleva a contemplar como parte de la reflexividad sobre la textualización los criterios que los alumnos tienen sobre la noción de párrafo.
}

82 Ernesto H. RODRÍGUEZ. Prácticas de revisión y edición de textos de alumnos bilingües... 
Considero que en algunos casos el autor reflexiona y toma decisiones de textualización basado, principalmente, en la revisión y valoración de sus propios recursos de escritura, ya que, tal como vemos en este caso, los comentarios del juez no le favorecen.

3) Versión inicial relacionada y final sin relación con el tema

Existen casos en que la versión inicial tiene relación con la lectura proporcionada y la versión final es un texto sin relación alguna con el tema. Cabe preguntarnos lo que sucedió en estos casos en relación a las decisiones del autor y la posible influencia de los comentarios del juez. Por ejemplo, en el texto tsim12 la alumna escribió en la versión inicial un comentario breve en el que retomaba algunos elementos de la lectura. El juez no hace sugerencias, simplemente hace un comentario breve y contundente sobre el pensamiento del hablante:

El pensamiento hablante tiene un poder muy importante en la conceptualizacion por que memoriza y clasifica (tsim12j)

Creo que este comentario motivó a la alumna a intentar hacer una reflexión sobre las características del pensamiento. Entonces ella se desvió de la lectura realizada y de su propia primera versión. La reflexividad sobre el tema y la intencionalidad para mejorar su escritura se manifiestan en su intento por manejar conceptos que la llevan a dificultades en la textualización relacionadas con la cohesión en el uso de pronombres para hacer referencia al tema que intentaba abordar, por ejemplo, al plantear al inicio de su segunda versión que:

Es importante conceptualizen muy bién entonces ya que sin el nosotros no podríamos memorizar y clasificar nuestros pensamientos, para ser transmitido por medio de nuestro lenguaje, y así poder hablar y entender a los demas y viceversa [...] (tsim12vi)

En contraste con el ejemplo anterior, la alumna se deja llevar por un comentario del juez que no le favorece para mejorar su texto, de manera tal que en no hace una reflexión sobre sus propios recursos, sino sobre una idea del juez que intenta desarrollar. Estos dos últimos ejemplos muestran que la reflexividad y las decisiones del autor para modificar su texto se deben en algunos casos a la influencia del juez y en otros exclusivamente a la revisión y valoración de los recursos propios de escritura. 


\section{Texto tsim12 completo}

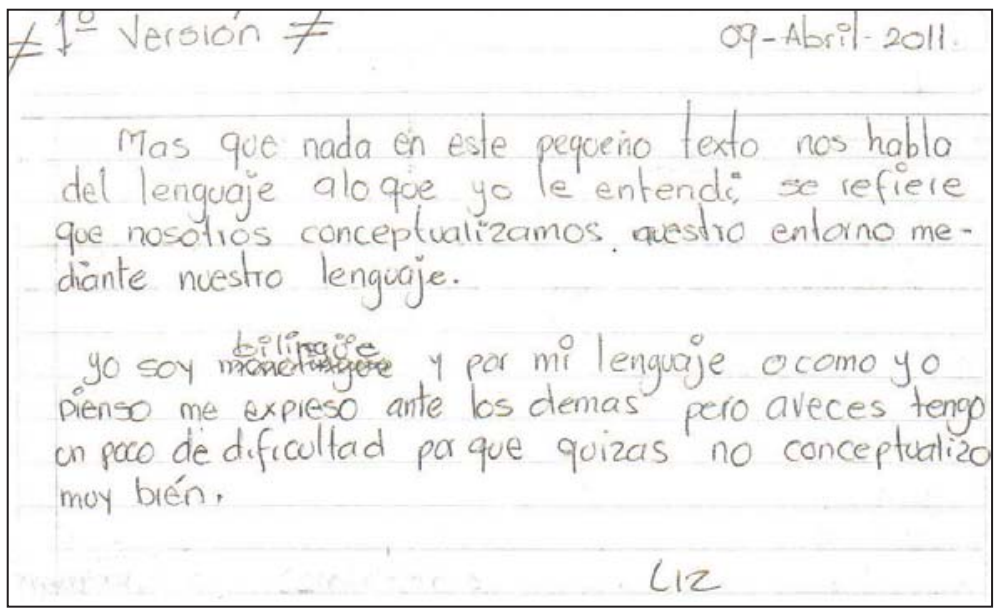

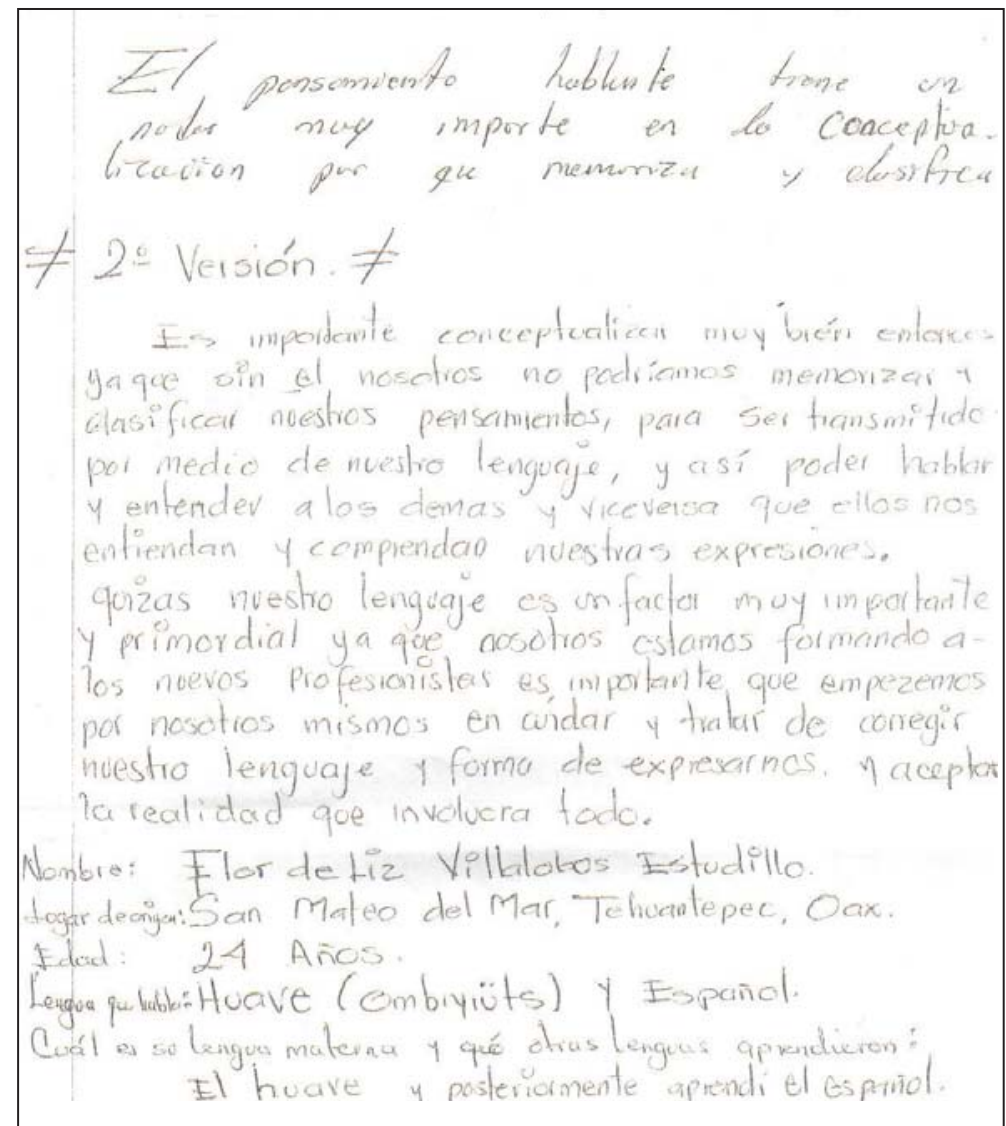

84 Ernesto H. RODRÍGUEZ. Prácticas de revisión y edición de textos de alumnos bilingües... 
4) Evidencias de intencionalidad para abordar el tema

Existe un conjunto de textos que con mayor claridad reflejan la intencionalidad del autor para abordar el tema en cuestión, sin embargo, el autor intenta pero no logra relacionar el tema abordado en la lectura, por diversas dificultades entre las que podemos destacar, por ejemplo, la falta de comprensión del texto, la confusión o los problemas de textualización enfrentados. El tema en cuestión aparece esbozado, mencionado o bien desarrollado con algunas dificultades, confusiones o interpretaciones de la lectura, mismas que son susceptibles de causar dificultades de comprensión a los lectores potenciales. En estos casos deseo estudiar la intencionalidad como un recurso y principio constitutivo y caracterizar aquello que el alumno intenta expresar, cómo lo hace y, qué solución da al enfrentar los diversos problemas de textualización que enfrenta. También contemplo la influencia de los comentarios del juez en la resolución de dificultades plasmadas en las segundas versiones. Por ejemplo, en el texto tsim13 la alumna aborda los conceptos presentes en la lectura proporcionada y redacta textos expositivos en las versiones inicial y final. Intenta poner orden en la exposición, pero tiene problemas para resolver aspectos vinculados con la comprensión de la lectura, la concordancia de género y para expresar la forma verbal cuando considera que "se entiende y se al mundo" y al hacer referencia a la actuación de un monolingüe y la manera en que capta y clasifica su alrededor, al explicar que la teoría

[...] se refiere a la manera en que una persona o varias utilizan la expresión, la forma de hablar de cada uno de ellos; la expresión se relaciona con la forma en que se entiende y se al mundo.

Ahora la hipótesis whorfiana fuerte da a entender que la persona que habla una sola lengua se expresa facilmente y capta con mayor facilidad lo que se encuentra a su alrededor y las clasifica. (tsim13vi)

El juez no hace sugerencias sobre estos aspectos ni sobre el género textual empleado, y simplemente asume que la autora debe completar la exposición pues apunta: "solo que falto el último subtema Evidencia empírica", y la autora en la segunda versión simplemente agrega este punto, donde una vez más intenta exponer y ejemplificar, pero se desvía del tema al considerar que la evidencia empírica "trata sobre los conocimientos previos a algo".

En otro caso la intencionalidad del autor para relacionar el tema puede ser influida por algún comentario del juez, por ejemplo en tsim 25 en la versión inicial del texto expositivo la alumna muestra evidencias de hacer un esfuerzo por referirse al tema, pero el juez, sin hacer sugerencias, hace un comentario que influye para que ella se desvíe de su primer intento y como resultado ella sigue la idea expresada por el juez, sin relación alguna con el tema ni con el intento que hizo en la primera versión. A continuación presento el comentario del juez. 
[...] que nosotros como seres humanos debemos de expresar nuestro sentir, pensamiento, y todo lo que en el suceda por medio de nuestro lenguaje, para asi poder hablar y entender nuestra realidad, para así poderlo llevar al campo semántico. (tsim25j)

Los jueces influyen a los autores a hacer modificaciones que en muchas ocasiones no favorecen que el autor mejore el texto, fundamentalmente cuando se desvían del tema que habían abordado o cuando cambian su comentario del texto en la primera versión por una exposición. Considero que algunos autores intentan desarrollar las temáticas escritas por los jueces, con el afán de demostrar su dominio de conceptos complejos en un ambiente académico, sin embargo, en el intento se desvían del tema de la lectura.

5) Principios de efectividad e intencionalidad sobre el género y el tema

Es fundamental caracterizar los textos en términos de la interrelación del género textual empleado y el tema de la lectura proporcionada. Además, es necesario contemplar el tipo de desempeño y avance en la redacción en términos del impacto de los comentarios de los jueces, de manera tal que sea posible determinar la importancia de la reflexividad en los procesos de revisión y edición de los textos, así como los principios constitutivos de efectividad, situacionalidad e intencionalidad (Beugrande e Dressler, 1997) y la reflexión sobre la textualización (Hyland, 2005). El análisis requiere revisar la relación entre género textual y tema en los textos que no cumplen las expectativas sobre el comentario de la lectura, pero que reflejan indudablemente evidencias sobre los procesos involucrados en la redacción y en la reflexividad presentes en la revisión y edición de los textos.

En la tabla 3 apreciamos el reducido número de textos conformados por comentario relacionado con el tema en versiones inicial (4 de 26) y final (2 de 26), de los cuales únicamente 2 mantienen estas características. Este es un dato relevante para reflexionar en torno a las concepciones de los estudiantes sobre los géneros textuales y la práctica de la redacción en ambientes académicos. 
Tabla 3 - El género textual y la relación con el tema de la lectura

\begin{tabular}{|c|c|c|c|c|c|}
\hline & $\begin{array}{l}\text { Comentario } \\
\text { relacionado } \\
\text { con el tema }\end{array}$ & $\begin{array}{l}\text { Comentario } \\
\text { sin relación } \\
\text { con el tema }\end{array}$ & $\begin{array}{l}\text { Exposiciones } \\
\text { relacionadas } \\
\text { con el tema }\end{array}$ & $\begin{array}{l}\text { Exposiciones } \\
\text { sin relación } \\
\text { con el tema }\end{array}$ & Total \\
\hline Versión inicial & $\begin{array}{c}4 \\
\text { (textos: 06, } \\
12,17,21)\end{array}$ & $\begin{array}{c}3 \\
\text { (textos: 09, 10, } \\
16)\end{array}$ & $\begin{array}{c}7 \\
\text { (textos:1, 2, 3, } \\
5,8,11,24)\end{array}$ & $\begin{array}{c}12 \\
\text { (textos:4, 7, 13, } \\
14,15,18,19,20, \\
22,23,25,26)\end{array}$ & 26 \\
\hline Versión final & $\begin{array}{c}2 \\
\text { (textos: } 17, \\
21)\end{array}$ & $\begin{array}{c}5 \\
\text { (textos: 07, 09, } \\
10,12,20)\end{array}$ & $\begin{array}{c}9 \\
\text { (textos:1,2,3, } \\
5,6,8,11,23 \\
24)\end{array}$ & $\begin{array}{c}10 \\
\text { (textos: } 4,13,14, \\
15,16,18,19,22, \\
25,26)\end{array}$ & 26 \\
\hline
\end{tabular}

No apreciamos un efecto favorable en el ejercicio en términos de la efectividad para lograr comentarios sobre la lectura en las versiones finales. Las variables a considerar pueden ser múltiples, tal vez la intervención de los jueces, la interpretación de las sugerencias o las propias decisiones de los autores. De hecho, los comentarios sobre la lectura disminuyen de la versión inicial ( 4 de 26 ) a la final ( 2 de 26 ) y, por otra parte, aumentan los comentarios sin relación con el tema de la versión inicial (3 de 26) a la final (5 de 26). Las exposiciones con y sin relación con el tema en las versiones iniciales y finales constituyen un número que no tiene cambios significativos, pues ligeramente avanzan al incorporar el tema (7vi a 9vf y 12vi a 10vf), pero la exposición es muy superior a los comentarios.

En el texto tsim17 la alumna cumple con el comentario relacionado con el tema y constituye un ejemplo representativo de mayor avance conforme a los principios regulativos de aceptabilidad, efectividad e intencionalidad. De hecho en su versión final hace cambios que favorecen la incorporación de información y de sus puntos de vista, además se posiciona ante el texto con precisión para plantear sus puntos de vista. A continuación presento algunos pasajes de este texto ${ }^{10}$.

Está hipótesis establece que existe una cierta relación entre las categorias gramaticales del lenguaje, que una persona habla y la manera de como ve y conceptualiza el mundo.

Creo que las dos hipótesis fuerte y debil tienen algo de razón, ya que en la primera podriamos decir que es cierto que la lengua de una persona monolingüe, dependiendo de su lengua índigena o el español, existen palabras que tienen un significado diferente y por lo tanto una conceptualización diferente de ver y expresar el mundo.

\footnotetext{
${ }^{10}$ Los pasajes que subrayo reflejan la intencionalidad de la alumna para enriquecer su texto y aportar elementos para completar información fundamental en la segunda versión.
}

Tellus, ano 11, n. 21, jul./dez. 2011 
[...] Estoy de acuerdo en que la lengua no determina la vision de lo que nos rodea si no que también la memoria es un factor importante para determinar el lenguaje. (tsim17vf)

El juez no aporta sugerencias concretas sobre el desempeño de la alumna, simplemente hace valoraciones, por ejemplo, al considerar que el texto tiene "mucha relación con la lectura", y que "hay que respetar los puntos y los guiones", a pesar de que en el escrito la alumna no presenta estos problemas. Este ejemplo refleja que a veces las observaciones del juez no resultan significativas para la edición de los textos que muestran un mayor avance, aceptabilidad, eficacia y propiedad.

Texto tsim17 completo

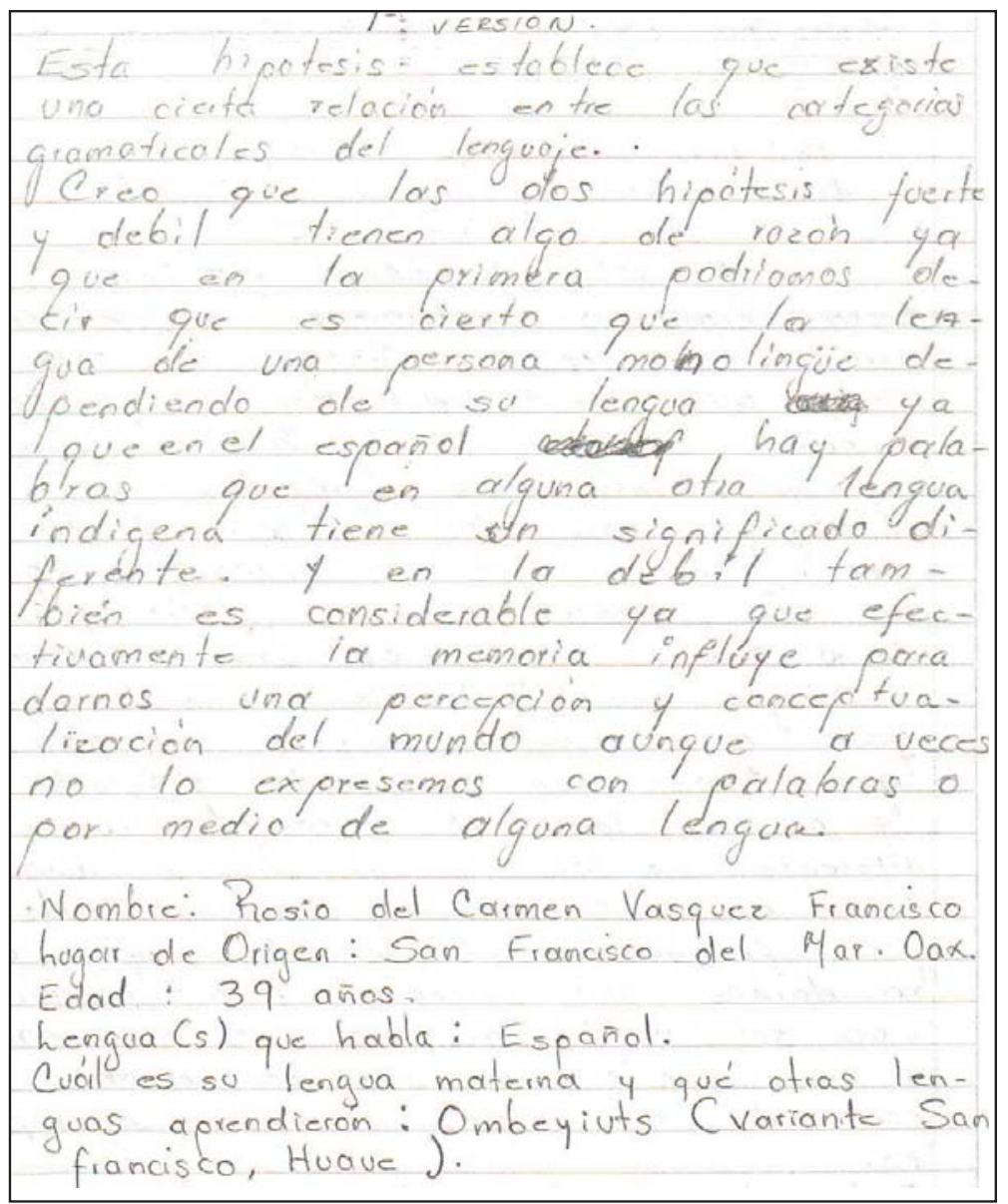

88 Ernesto H. RODRÍGUEZ. Prácticas de revisión y edición de textos de alumnos bilingües... 


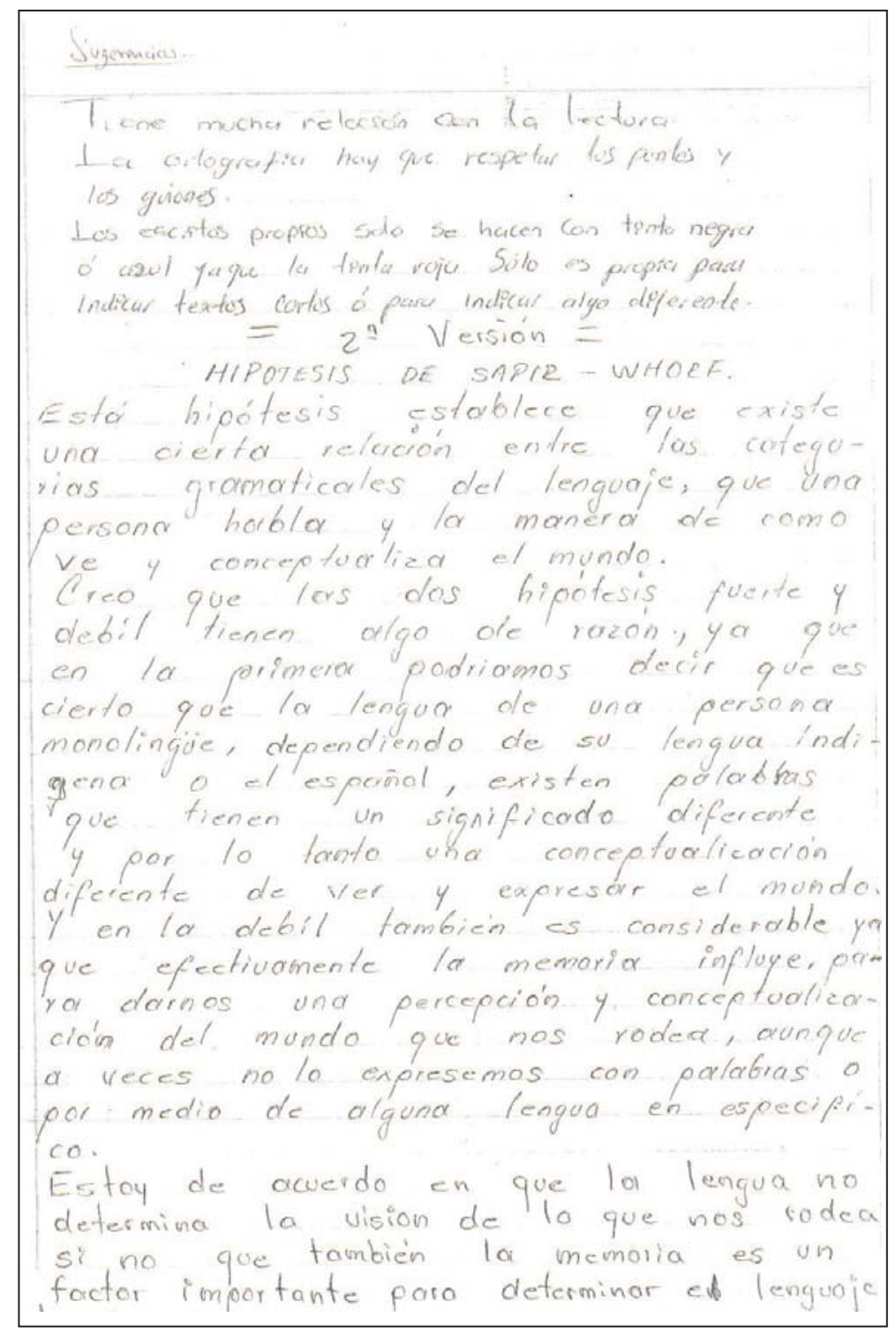

\section{Sugerencias de los jueces y los cambios en las segundas versiones}

Para hacer el análisis y dar seguimiento a los comentarios de los jueces y las decisiones de textualización en las segundas versiones presento en la tabla 4 el tipo de intervención que hacen 22 alumnos al asumir el papel de juez para comentar o sugerir. Esta información me permite contemplar la manera en que esta intervención escrita puede o no impactar o influir en las decisiones 
del autor para revisar y editar su texto en la segunda versión. Además, en la tabla 5 presento el tipo de decisiones y modificaciones que realizó el autor en la segunda versión. Así, es posible establecer correlaciones más precisas entre las sugerencias, las decisiones en la revisión y la edición. En esta ocasión presento algunos ejemplos significativos. Revisemos ambas tablas.

Tabla 4 - Sobre el tipo de sugerencias y comentarios de los jueces.

\begin{tabular}{|l|c|}
\hline Sugerencias u opiniones del juez al autor & $\begin{array}{l}\text { Apariciones en 22 textos } \\
\text { (En } 4 \text { de los 26 textos no } \\
\text { aparecen comentarios del juez) }\end{array}$ \\
\hline Sugiere sobre la cohesión & 5 (textos: 04, 17, 18, 21 y 23) \\
\hline $\begin{array}{l}\text { Sugiere que explique con sus propias palabras, } \\
\text { expresar su opinión. }\end{array}$ & 5 (textos: 05, 08, 14,19 y 20) \\
\hline $\begin{array}{l}\text { Sugiere que el autor proporcione información de } \\
\text { la lectura, que aborde el tema, que sea coherente, } \\
\text { que lea primero }\end{array}$ & $\begin{array}{c}9 \text { (textos: 04, 06, 07, 13, 14, 15, } \\
16,19 \text { y 26) }\end{array}$ \\
\hline $\begin{array}{l}\text { Sugiere que incorpore información para } \\
\text { enriquecer el texto }\end{array}$ & 2 (textos: 13 y 19) \\
\hline $\begin{array}{l}\text { Expresa punto de vista sobre la lectura, el tema o } \\
\text { las ideas del autor, pero no sugiere }\end{array}$ & 5 (textos: 01, 09, 10, 25, 26) \\
\hline $\begin{array}{l}\text { Aporta información sobre el tema pero no sugiere } \\
\text { nada }\end{array}$ & 5 (textos: 12, 21 y 23) \\
\hline Aporta información ajena al tema & 3 (textos: 02, 10, 16) \\
\hline Elogia la redacción & 2 (textos: 18 y 21) \\
\hline Señala lo que no pudo hacer el autor & \\
\hline
\end{tabular}

La tabla 4 muestra que en general los jueces se centran predominantemente en el manejo de información, ya sea relacionada o ajena al tema, en algunos casos hacen sugerencias y en otros simplemente aportando información o bien opiniones sin relación alguna. El hecho de que sólo 5 de 22 alumnos sugieran que el autor exprese su opinión con sus propias palabras, indudablemente refleja que, en su mayoría, para los jueces la redacción del comentario crítico no es un aspecto fundamental. 
Tabla 5 - Tipo de modificaciones en la segunda versión

\begin{tabular}{|c|c|}
\hline $\begin{array}{l}\text { Sobre el tipo de decisiones para modificar } \\
\text { la segunda versión }\end{array}$ & Apariciones en los 22 textos \\
\hline No realiza cambios significativos & 6 (textos: $01,02,04,05,09,17)$ \\
\hline Cambia género textual & 4 (textos: 07, 20 y 06, 16) \\
\hline Cambia contenido de 1a versión inicial & 6 (textos: $12,16,19,20,25,26)$ \\
\hline Incorpora información & 5 (textos: $13,14,19,21$ y 23 ) \\
\hline Elimina información & 3 (textos: 08,15 y 18 ) \\
\hline $\begin{array}{l}\text { Se apropia o se influye de las ideas } \\
\text { del juez y las sigue en su escrito }\end{array}$ & 3 (textos: 10, 12, 25) \\
\hline Segunda versión es totalmente distinta & 5 (textos: 16, 19, 20, 25, 26) \\
\hline Modifica puntuación, ortografía & 2 (textos: 17,21$)$ \\
\hline Responde al comentario del juez & 2 (textos? 01, 07) \\
\hline
\end{tabular}

La tabla 5 nos muestra el predominio de la toma de decisiones relacionadas con el manejo de la información en el cambio de contenidos, la incorporación de información. El cambio de género textual se limita a 4. Podemos constatar que la mayoría de autores y jueces no contemplan el cambio del género expositivo al comentario.

Anteriormente, presenté el caso en que el autor mejora su texto en la segunda versión (tsim17vf), a pesar de que el juez no hace sugerencias que le ayuden a esta mejoría. En otros casos la intervención del juez aporta elementos para que el autor escriba una segunda versión mejorada. Estos textos son relevantes para estudiar las decisiones de los autores y el impacto de las sugerencias, sin embargo, también es necesario estudiar la intencionalidad de aquellos alumnos que se enfrentan a la problemática de redacción y la manera en que dan solución a las dificultades, a pesar de que su texto no muestre un avance o mejoría respecto a la versión anterior. Por ello importa hacer una caracterización desde la perspectiva de la intencionalidad y la reflexividad, contemplando los comentarios de los jueces. De esta manera podemos considerar los principios constitutivos y regulatorios que intervienen en la redacción.

Un caso en el que el autor del texto escribe una versión totalmente distinta a su primer escrito debido a la influencia del juez aparece en el texto tsim25. El juez no se refiere al contenido escrito por el autor y expresa su punto de vista sobre aspectos relacionados con el tema, pero no sugiere nada. En su primera versión el autor escribió un texto expositivo que informa sobre la hipótesis Sapir-Whorf y el juez opina

[...] que nosotros como seres humanos debemos de expresar nuestro sentir, pensamiento, y todo lo que en el suceda por medio de nuestro lenguaje, para asi poder hablar y entender nuestra realidad, para así poderlo llevar al campo semántico. (tsim25j) 
Ante esta opinión el autor escribe un texto totalmente distinto a su primera versión, intentando recuperar aspectos tomados de la lectura y, principalmente, tomando algunas de las ideas del juez. Intenta incorporar conceptos, pero no lo logra, puesto que se desvía del tema abordado en la primera versión.

La forma de expresarnos por nosotros mismo en el lenguaje, el hablante sigue la forma de entender y conceptualizar, memorizar y determinar la lengua. [...] (tsim25vf).

Existen casos en que los comentarios del juez son determinantes para que el autor modifique el texto incorporando aspectos relevantes, a pesar de que la intencionalidad del autor no se vea reflejada en una segunda versión que cumpla los criterios de aceptabilidad y propiedad, como apreciamos en tsih19, donde, tal como presenté anteriormente, el autor escribe una primera versión sin relación alguna con el tema de la lectura, al escribir, por ejemplo, que "Yo le entendí al texto que una persona habla y expresa sus sentimientos de acuerdo a su manera de como percibe el mundo que lo rodea". Ante esta situación el juez hace una recomendación sobre la manera de estructurar las ideas para mejorar el texto.

[...] *En el texto menciona dos hipótesis sería bueno que dieras tu punto de vista de cada una y al final dar una conclusión de ambas (tsih19j).

El autor intenta mejorar su texto y en la versión final modifica totalmente lo que antes escribió y hace un esquema de las dos hipótesis, pero no hace el comentario ni la conclusión que le sugirió el juez. Es decir, asume los cambios que debe hacer, intenta resolver la problemática y da una solución parcial al problema enfrentado. Por el contrario, un ejemplo en el que el comentario del juez parece ser determinante para que el autor mejore su texto aparece en tsih07, donde, como recordamos, el juez le dice al autor que "no te diste el tiempo de leer el texto" y "que debes ser un poquito mas crítico con tígo". Ante este comentario el autor modifica el texto expositivo sin relación con el tema de la lectura y en la segunda versión escribe un comentario que intenta relacionar con el tema, aunque no lo logra. Además se posiciona incluyéndose en segunda persona del plural para expresar su punto de vista.

De acuerdo a la lectura que se estuvo analizando es muy importante que nosotros nos demos cuenta con que personas estamos hablando para darnos cuenta quienes son si son de la misma comunidad porque muchas veces pasa eso que cuando un monolingüe va a otro lugar [...] porque hay palabras que si coinciden pero sus significados no los mismos son diferentes (tsih07vf).

Indudablemente, este ejemplo manifiesta la intencionalidad en un proceso reflexivo sobre el género textual y los recursos para hacer un comentario, a pesar de no haber logrado abordar el tema de la lectura. A continuación presento el texto completo.

92 Ernesto H. RODRÍGUEZ. Prácticas de revisión y edición de textos de alumnos bilingües... 


\section{Texto tsih07 completo}

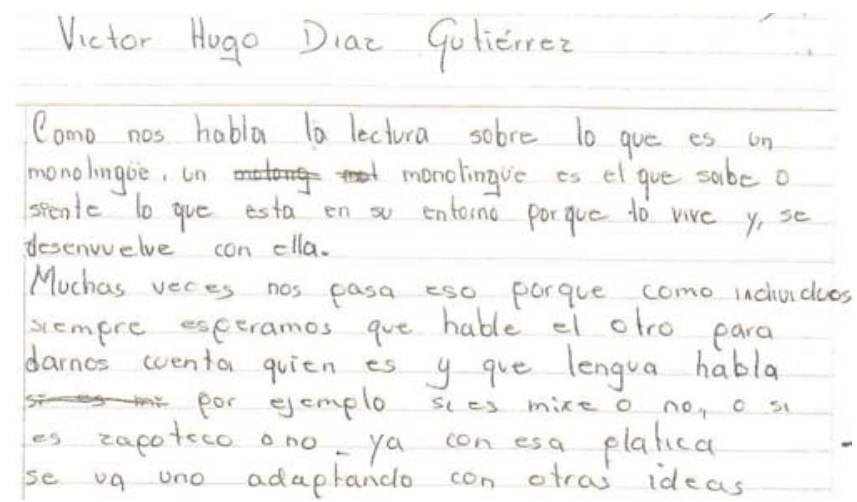

\section{Juez}

Por lo qe note, no te diste el trempo de leer el textb, no tie preocupes a la mayoría nos paso. "no es cierto"

Pienso que debes ser un poquito mas critico con figo.

De acuerdo a la lectura que se estuvo analizando es muy importante que nosotros nos demos cuenta con que personas estamos hablando para darnos cuenta quienes son st son de la misma comunidad forque muchas veces pasa eso que cuando un monolingüe. vo a otro lugar $y$ se encuentra con uno que no habla la misma lengua por eso es muy importante ver donde andamos quienes somos con que nos identificamos y también como manejumos nuestra lenqua que lerminos usamos, porque hay palabras que si coinciden pero sus significados no los mismos son diferentes, a lo mejor si hay palabras que coinciden pero son palabras que cambian nadamas del sonido porque si son de la misma region pero de diferentes comunidades

* Nombre: Victor Hugo Díaz Gutiérrez

* Lugar de origen: Santa Maria Tlahuitoltepec Mixe. 0

* Edad: 22 años

* Lenqua(s)o que habla: Mixe, Espoñol. * Cual es su lenqua Materna y que otras lenguas. 


\section{Recapitulación}

En la revisión y edición de textos donde intervienen los alumnos como jueces existen diversas prácticas interactivas sobre la construcción de procesos de textualización, regulación de la expresión escrita y retroalimentación en la valoración de la escritura de un compañero. Podemos percatarnos de que los autores no se encuentran habituados a este tipo de prácticas de redacción académica y deben tomar decisiones para resolver diversas problemáticas vinculadas con la cohesión, la coherencia, los estándares socializados de informatividad, situacionalidad y aceptabilidad, y por otra parte, los principios regulativos de eficacia, efectividad y propiedad. La intencionalidad y la reflexividad son elementos fundamentales que dan vida a la comunicación.

Cada alumno enfrenta y resuelve problemáticas de manera diferente al valorar sus propios escritos y las opiniones de los jueces para tomar decisiones de textualización. En su mayoría enfrentaron dificultades para establecer la distinción entre un texto expositivo y un comentario, así como para escribir sobre el tema de la lectura proporcionada, en algunos casos por dificultades con la compresión de la lectura y en otros por la decisión de opinar sobre temas relacionados o bien distintos al tema. El estudio me permitió caracterizar los recursos y repertorios de textualización de los estudiantes y, principalmente, el tipo de decisiones para la revisión y edición de los escritos al contemplar la retroalimentación escrita del juez. Además, determiné la naturaleza de las sugerencias que hacen los jueces y la manera en que los autores las valoran y, en su caso, consideran para la revisión y edición de su texto.

Los jueces opinan desde diversas perspectivas, por ejemplo, unos hacen sugerencias concretas, otros simplemente hacen comentarios ajenos al tema y no sugieren nada. Predominan los comentarios y las sugerencias sobre las ideas, contenidos y puntos de vista. En general los jueces no abordan aspectos sobre recursos de cohesión que involucran cuestiones de la gramática básica del español, a pesar de que en los textos existen problemas de referencia pronominal y concordancia. Los autores reaccionan de manera diversa, unos autores contemplan lo planteado por el juez y modifican, por ejemplo, el género, el tema de la primera versión, la estructura interna, eliminan pasajes o bien hacen un texto totalmente diferente, otros ignoran los comentarios. Existen casos en que los jueces hacen comentarios o aportan información ajena al tema y los autores se apropian de estas ideas y las plasman en sus textos; así la segunda versión pierde calidad respecto al primer escrito. Por el contrario, existen textos que mejoran a pesar de que el juez no aportó elementos para dicha mejoría. En la valoración de los comentarios del juez los autores contemplan recursos regulatorios que involucran la reflexividad sobre su actuación, así como criterios de aceptabilidad, eficacia y propiedad. La reflexividad incluye

94 Ernesto H. RODRÍGUEZ. Prácticas de revisión y edición de textos de alumnos bilingües... 
además, de la valoración sobre la pertinencia del comentario de los jueces, la toma de decisiones sobre los recursos de redacción plasmados en la primera versión y la manera de modificar el texto.

La perspectiva de este estudio es la caracterización de recursos de redacción, la reflexividad sobre la práctica de textualización y contemplar la intencionalidad en la expresión escrita para comprender la manera en que los estudiantes enfrentan las problemáticas de redacción y cuáles son los aspectos que, al asumir el papel de jueces, consideran relevantes para hacer sugerencias al autor, y las valoraciones sobre los comentarios del los jueces y decisiones de los autores para elaborar la segunda versión.

\section{Bibliografía}

ADAM, Jean-Michel. La linguistique textuelle: introduction á l'analyse textuelle des discours. Paris: Armand Colin, 2005.

BEUGRANDE, Robert; DRESSLER, Wolfangr. Introducción a la lingüística del texto. Barcelona: Ariel Lingüística, 1997.

BÉGUELIN, Marie-José. Unidades de lengua y unidades de escritura. Evolución y modalidades de la segmentación gráfica. En: FERREIRO, Emilia (Comp.). Relaciones de (in)dependencia entre oralidad y escritura. Barcelona: Gedisa, 2002.

BLANCHE-BENVENISTE, Claire. Estudios lingüísticos sobre la relación entre oralidad y escritura. Barcelona: Gedisa, 1998.

BRICE HEATH, Shirley. Ways with words: language, life, and work in communities and classrooms. New York: Cambridge University Press, 1983.

BRICE HEATH, Shirley; STREET, Brian (com MILLS, Molly). Ethnography: approaches to language and literacy. New York: Teachers College Press, 2008.

CAMPS, David. Advanced EFL student's revision practices through their writing process. En: CARTER, Awena; LILLIS, Theresa; PARKIN, Sue. Why writing matters: issues of access and identity in writing research and pedagogy. Philadelphia: John Benjamins Publishing Company, 2009.

CIFUENTES, Bárbara, Letras sobre voces: multilingüismo a través de la historia. México: Ciesas-INI, 1998.

HALLIDAY, Michael; HASAN, Rugaiya. Cohesion in English. London: Longman, 1976.

HERNÁNDEZ RODRÍGUEZ, Ernesto. La redacción de textos con intencionalidad argumentativa de alumnos de bachillerato. Tesis (Maestría en Ciencias con Especialidad en Investigaciones Educativas. Asesora Dra. Emilia Ferreiro) - Centro de Investigación y de Estudios Avanzados del Instituto Politécnico Nacional, México, 2003.

HYLAND, Ken. Metadiscourse: exploring interaction in writing. London: Continuum, 2005. 
LEA, Mary. Academic literacies and learning in higher education: constructing knowledge through texts and experience. En: JONES, Carys; TURNER, Joan; STREET, Brian (Eds.). Students writing in the university: cultural and epistemological issues. Philadelphia: John Benjamins Publishing Company, 1999.

LEA, Mary; STREET, Brian V. Student writing in higher education: an academic literacies approach. Studies in Higher Education, v. 23, n. 2, p. 157-172, 1998.

LILLIS, Theresa M. Student writing: access, regulation, desire. London: Routledge, 2001.

MANRIQUE CASTAÑEDA, Leonardo. Atlas cultural de México: lingüística. México: SEP-INAH-Planeta, 1988.

RECANATI, Francois. La transparencia y la enunciación: introducción a la pragmática. Buenos Aires: Hachette, 1979.

. Literal meaning. Cambridge: Cambridge University Press, 2004.

SCOTT, Mary. Agency and Subjectivity in Student writing. En: JONES, Carys; TURNER, Joan; STREET, Brian (Eds.). Students writing in the university: cultural and epistemological issues. Philadelphia: John Benjamins Publishing Company, 1999.

STREET, Brian V. The future of 'social literacies'. En: BAYNHAM, Mike; PRINSLOO, Mastin (Eds.). The future of literacy studies. Hampshire: Palgrave Macmillan, 2009a.

. "Hidden" Features of Academic Paper Writing. Working papers in educational linguistics, v. 24, n. 1, 2009b.

WHORF, Benjamin Lee. Language, thought and reality. New York: The MIT Press, 1964.

ZAVALA, Virginia. “¿Quién está diciendo eso?” Literacidad académica, identidad y poder en la educación superior. En: KALMAN, Judith; STREET, Brian. Lectura, escritura y matemáticas como prácticas sociales: diálogos con América latina. México: Siglo XXI-CREFAL, 2009.

Recebido em 17 de maio de 2011 Aprovado para publicação em 15 de junho de 2011

96 Ernesto H. RODRÍGUEZ. Prácticas de revisión y edición de textos de alumnos bilingües... 